\title{
ROLE OF COPY NUMBER VARIANTS IN ADHD
}

Investigators from Brazil determined if copy number variants (CNVs) in glutamate metabotropic receptor genes (GRM) were overrepresented in 1038 individuals with ADHD compared to 1057 subjects without ADHD. No significant difference in the total number of $\mathrm{CNV}$ s was found in the two population samples $(\mathrm{P}=0.326)$. The presence of CNVs was associated with lower IQ scores in ADHD samples $(\mathrm{P}=0.026)$ but not in the sample without ADHD. CNVs in GRM5 were associated with anxiety disorders in ADHD cases $(\mathrm{P}=0.002)$, but not in subjects without ADHD. CNVs in the glutamatergic genes were associated with cognitive and clinical characteristics of ADHD. (AkutagavaMartins GC, et al. Glutamatergic copy number variants and their role in attentiondeficit/hyperactivity disorder. Am J Med Genet B Neuropsychiatr Genet 2014 Jul 2).

COMMENTARY. The results of this study suggest a role for glutamate in ADHD. In an investigation of the association between metabotropic glutamate receptor subtype 7-gene polymorphism and treatment response to methylphenidate, children with the G/A genotype had a more pronounced response rate than children with the $\mathrm{G} / \mathrm{G}$ genotype [1].

\section{References.}

1. Park S, et al. J Child Adolesc Psychopharmacol. 2014 May;24(4):223-7.

\section{DIAGNOSTIC CRITERIA FOR ADHD}

\section{IMPACT OF THE DSM-5 CRITERIA ON PREVALENCE OF ADHD}

Investigators at the National Institute of Mental Health, Bethesda, MD, compared the prevalence and clinical correlates of DSM-IV-TR versus DSM-5-defined ADHD and subtypes in a nationally representative sample of US youth based on age-of-onset criterion. Extension of the age-of-onset criterion from 7 to 12 years led to an increase in the prevalence rate of ADHD from 7.38\% (DSM-IV-TR) to 10.84\% (DSM-5). Severity of ADHD and patterns of comorbidity were not changed by the later age-of-onset, but the group with later age of onset was more likely to be from lower income and ethnic minority families. (Vande Voort JL, He JP, Jameson ND, Merikangas KR. Impact of the DSM-5 attention-deficit/hyperactivity disorder age-of-onset criterion in the US Adolescent population. J Am Acad Child Adolesc Psychiatry 2014 Jul;53(7):736-44).

COMMENTARY. The DSM-5 edition released in May 2013 replaces the DSMIV-TR edition and the changes are as follows: symptoms can now occur by age 12 rather than by age 6 , and for adults and adolescents age 17 or older, only 5 symptoms are needed instead of the 6 needed for younger children.

Unchanged is the requirement that symptoms must be present for at least 6 months and they are inappropriate for developmental level; several symptoms were present before age 12 years; several symptoms are present in two or more settings (e.g., at home, school or work; with friends or relatives; in other activities); symptoms interfere with or reduce the quality of social, school, or work; and the symptoms are not better 